\title{
Absorção de nutrientes da soja sob níveis de compactação
}

\author{
Soybean nutrient uptake under compaction levels \\ Absorción de nutrientes de la soja en niveles de compactación
}

Recebido: 18/11/2021 | Revisado: 24/11/2021 | Aceito: 25/11/2021 | Publicado: 05/12/2021

\author{
Marina Fabris Matté \\ ORCID: https://orcid.org/0000-0002-3799-8260 \\ Universidade Estadual do Oeste do Paraná, Brasil \\ E-mail:mfmagroo@gmail.com \\ Claudio Yuji Tsutsumi \\ ORCID: https://orcid.org/0000-0003-2455-7279 \\ Universidade Estadual do Oeste do Paraná, Brasil \\ E-mail: claudio.tsutsumi@unioeste.br \\ Deonir Secco \\ ORCID: https://orcid.org/0000-0002-3042-159X \\ Universidade Estadual do Oeste do Paraná, Brasil \\ E-mail: deonir.secco@unioeste.br \\ Doglas Bassegio \\ ORCID: https://orcid.org/0000-0001-6628-8594 \\ Universidade Estadual do Oeste do Paraná, Brasil \\ E-mail: doglas.bassegio@unioeste.br
}

\begin{abstract}
Resumo
A preocupação com a compactação do solo vem aumentando devido ao elevado tráfego de máquinas e equipamentos em sistema de plantio direto. Com isto, tem-se a baixa infiltração da água, o que afeta a dinâmica dos nutrientes no solo. Com isso, o objetivo do trabalho foi avaliar a absorção de nutrientes da soja sob níveis de compactação do solo $\left(1,1 ; 1,2 ; 1,3 ; 1,4\right.$ e $\left.1,5 \mathrm{Mg} \mathrm{ha}^{-1}\right)$. O trabalho foi conduzido em ambiente protegido na Universidade Estadual do Oeste do Paraná, Campus de Cascavel, em delineamento inteiramente ao acaso, com cinco repetições. O experimento foi montado com anéis de PVC sobrepostos, com $5 \mathrm{~cm}, 10 \mathrm{~cm}$ e $20 \mathrm{~cm}$ de altura. O anel de PVC de $20 \mathrm{~cm}$ continha solo sem compactação. Sobre esses foi disposto o anel de PVC de $5 \mathrm{~cm}$ com os níveis de compactação e, por último, acima de todos, o terceiro anel de PVC de $10 \mathrm{~cm}$ com solo sem compactação. A absorção de nutrientes pelas plantas de soja foi analisada na fase de desenvolvimento R1. A compactação do solo induziu alterações na absorção e concentração de $\mathrm{P}, \mathrm{K}$ e $\mathrm{Mg}$ nas plantas de soja. Foi observado acréscimo nos teores de $\mathrm{P}$ e $\mathrm{K}$ e redução do $\mathrm{Mg}$ com o aumento da compactação, o que foi devido ao maior contato entre solo e raiz pelo confinamento das raízes na camada superior do solo compactado.
\end{abstract}

Palavras-chave: Glycine max; Compactação; Nutrientes.

\begin{abstract}
The concern with soil compaction has increased due to the high traffic of machinery and equipment in a no-tillage system. With this, there is low water infiltration, which affects the dynamics of nutrients in the soil. Thus, the objective of this work was to evaluate the uptake of soybean nutrients under soil compaction levels $(1.1 ; 1.2 ; 1.3 ; 1.4$ and $\left.1.5 \mathrm{Mg} \mathrm{ha}^{-1}\right)$. The work was carried out in a protected environment at the State University of West Paraná, Cascavel Campus, in a completely randomized design, with five replications. The experiment was set up with overlapping PVC rings, $5 \mathrm{~cm}, 10 \mathrm{~cm}$ and $20 \mathrm{~cm}$ in height. The $20 \mathrm{~cm}$ PVC ring contained uncompacted soil. Over these, the $5 \mathrm{~cm}$ PVC ring with the compaction levels was placed and, lastly, above all, the third $10 \mathrm{~cm}$ PVC ring with uncompacted soil. Nutrient uptake by soybean plants was analyzed in the R1 development phase. Soil compaction induced alterations in the uptake and concentration of $\mathrm{P}, \mathrm{K}$ and $\mathrm{Mg}$ in soybean plants. An increase in $\mathrm{P}$ and $\mathrm{K}$ contents was observed with increasing compaction, which was due to the greater contact between soil and root due to the confinement of the roots in the upper layer of compacted soil.
\end{abstract}

Keywords: Glycine max; Compaction; Nutrients.

\section{Resumen}

La preocupación por la compactación del suelo ha aumentado debido al alto tráfico de maquinaria y equipo en un sistema de labranza cero. Con esto, existe una baja infiltración de agua, lo que afecta la dinámica de los nutrientes en el suelo. Así, el objetivo de este trabajo fue evaluar la absorción de nutrientes de la soja bajo niveles de compactación del suelo $\left(1.1 ; 1.2 ; 1.3 ; 1.4\right.$ y $\left.1.5 \mathrm{Mg} \mathrm{ha}^{-1}\right)$. El trabajo se realizó en un ambiente protegido en la Universidad Estatal de West Paraná, Campus Cascavel, en un diseño completamente al azar, con cinco réplicas. El experimento se organizó con anillos de PVC superpuestos, de $5 \mathrm{~cm}, 10 \mathrm{~cm}$ y $20 \mathrm{~cm}$ de altura. El anillo de PVC de $20 \mathrm{~cm}$ contenía tierra sin 
compactar. Sobre estos se colocó el anillo de PVC de $5 \mathrm{~cm}$ con los niveles de compactación y, por último, sobre todo, el tercer anillo de PVC de $10 \mathrm{~cm}$ con suelo sin compactar. La absorción de nutrientes por las plantas de soja se analizó en la fase de desarrollo R1. La compactación del suelo indujo cambios en la absorción y concentración de $\mathrm{P}, \mathrm{K}$ y Mg en plantas de soja. Se observó un aumento en los contenidos de P y K al aumentar la compactación, lo que se debió al mayor contacto entre suelo y raíz por el confinamiento de las raíces en la capa superior del suelo compactado.

Palabras clave: Glycine max; Compactación; Nutrientes.

\section{Introdução}

A soja é um cereal de grande relevância no mercado econômico brasileiro, pois além de ser utilizado como farelo para o trato de animais, o óleo extraído dessa semente é utilizado em distintas disposições finais. Em seu processo de desenvolvimento, a planta da soja busca boa nutrição mineral para maior produção de sementes, tornando seu cultivo altamente viável, pois a fertilidade é um pilar fundamental para maior produtividade, buscando rentabilidade final de colheita.

Uma das principais limitações ao aumento de produtividades das plantas é a limitação física dos solos cultivados, mais conhecida como compactação (Costa et al., 2009, Tokura et al., 2021). Como principais causas da compactação de solo é válido citar o tráfego intenso de maquinários agrícolas, a modernização e mal o preparo do solo (Ren et al., 2019). Com a modernização da agricultura, o aumento no tamanho e no peso dos maquinários, houve a intensificação da compactação do solo (Bergamin et al., 2010; Spliethoff et al., 2020; Bareta Junior et al., 2021).

Na camada compactada, há alteração no balanço entre macro e microporos e na porosidade total do solo, influenciando o espaço destinado ao crescimento radicular e área explorada de solo pelas raízes (Beutler e Centurion, 2004; Bergamin et al., 2010). A consequência direta da compactação do solo é a inibição do desenvolvimento das raízes nas camadas mais profundas, da absorção dos nutrientes essenciais e da água. Além disso, ocorre a menor aeração do solo e a má infiltração da água das chuvas, fazendo com que essa escoe superficialmente causando erosões. Todos esses fatores acarretam o subdesenvolvimento das plantas e, por consequência, a redução da produtividade final.

A compactação influencia na absorção dos nutrientes, no crescimento da parte aérea e do sistema radicular, variando de acordo com a espécie, com a classificação do solo e com a quantidade de água nele existente (Queiroz-Voltan et al., 2000, Medeiros et al., 2005). Assim, reduz-se a disponibilidade dessa água e de nutrientes para as plantas, definindo que a compactação interfere na produtividade temporariamente ou de forma permanente. Com esse problema, o fluxo de massa é reduzido, afetando assim a transpiração das plantas que, ao chegar em certo ponto, pode provocar uma interferência na difusão dos nutrientes, processos responsáveis pelo transporte de nutrientes até às raízes (Silva et al., 2008).

O objetivo do trabalho foi avaliar a absorção de nutrientes da soja sob níveis de compactação do solo.

\section{Metodologia}

O trabalho foi conduzido em ambiente protegido na Universidade Estadual do Oeste do Paraná, Campus de Cascavel, Cascavel - PR. O clima da região, segundo a classificação de Köppen, é do tipo Cfa, Subtropical Úmido Mesotérmico, com precipitação média anual superior a $1500 \mathrm{~mm}$, verões quentes, com tendência de concentração das chuvas (temperatura média superior a $22^{\circ} \mathrm{C}$ ), invernos com geadas pouco frequentes (temperatura média inferior a $18^{\circ} \mathrm{C}$ ), sem estação seca definida (Caviglione et al., 2000).

O solo utilizado no experimento foi retirado de lavoura comercial na cidade de Corbélia (Latitude $24^{\circ} 48^{\prime} 49,78^{\prime \prime} \mathrm{S}$ e Longitude: $53^{\circ} 16^{\prime} 42,51^{\prime \prime}$ O). As análises químicas do solo antes do plantio e após a colheita, e a análise física foram realizadas encontram-se na Tabela 1. O solo utilizado no experimento tem como classificação morfológica Latossolo, por ser um solo altamente intemperizado, velho e profundo, chegando a mais de dois metros de espessura. 
Tabela 1. Caracterização química do solo.

\begin{tabular}{|c|c|c|c|c|c|c|c|c|c|}
\hline \multirow[t]{2}{*}{$\mathrm{pH}$} & $\mathrm{P}$ & $\mathrm{C}$ & $\mathrm{Al}^{-3}$ & $\mathrm{H}+\mathrm{A}$ & $\mathrm{Mg}$ & K & SB & $\mathrm{T}$ & \multirow{2}{*}{$\begin{array}{l}\mathrm{V} \\
\% \\
\end{array}$} \\
\hline & $\mathrm{Mg} \mathrm{dm}^{-3}$ & \multicolumn{7}{|c|}{ - } & \\
\hline 4,4 & 55,91 & 4,23 & 0,57 & 8,36 & 1 & 0,59 & 5,82 & 14,18 & 41,04 \\
\hline
\end{tabular}

SB-Soma das bases; T - Capacidade de troca de cátions; V - Saturação por bases. Fonte: Autores.

Com base nos resultados da análise de solo, foram realizadas as correções necessárias para plantio. A formulação do adubo utilizado foi a 04-24-16 Topmix+micro®. A cultivar de soja escolhida foi a ND 5909.

O experimento foi montado com anéis de PVC com $5 \mathrm{~cm}, 10 \mathrm{~cm}$ e $20 \mathrm{~cm}$ de altura. O anel de PVC de $20 \mathrm{~cm}$ continha solo sem compactação. Sobre esses foi disposto o anel de PVC de $5 \mathrm{~cm}$ com solo compacto e, por último, acima de todos, o terceiro anel de PVC de $10 \mathrm{~cm}$ com solo sem compactação. Todos os anéis foram unidos por fita adesiva.

Para a compactação do solo no anel de PVC de 5 centímetros de altura, foram utilizados um martelo de ferro, a prancha de madeira circular e, quando necessário, a água para auxílio na compactação. Golpes com o martelo foram efetuados na prancha de madeira até atingir a compactação desejada, como realizado por Silva e Rosolem (2002).

O plantio ocorreu em 18 de outubro de 2017, obedecendo o seguinte procedimento: no terceiro anel de PVC de $10 \mathrm{~cm}$, contendo o solo não compactado, foram semeadas 6 sementes de soja e, paralelo a essas, 5 g de adubo. Já no anel de PVC de $20 \mathrm{~cm}$ foram misturados ao solo não compactado 10 gramas do adubo. A irrigação ocorreu 3 vezes por semana, em volume de $200 \mathrm{ml}$ por vaso. Não foi aplicado nenhum tipo de defensivo agrícola nas plantas de soja.

A análise foliar foi realizada na fase de desenvolvimento R1 (Figura 1). Foram coletados 35 trifólios por talhão, com pecíolo, sendo esse retirado no processo de secagem das folhas. Após a colheita, as folhas passaram por processo de secagem. Os trifólios foram dispostos em pacotes de papel individualizados, conduzido em estufa de esterilização e secagem, com temperatura de $65^{\circ} \mathrm{C}$ até atingir peso constante $(72 \mathrm{~h})$.

Figura 1. Plantas de soja com 38 dias após a semeadura.

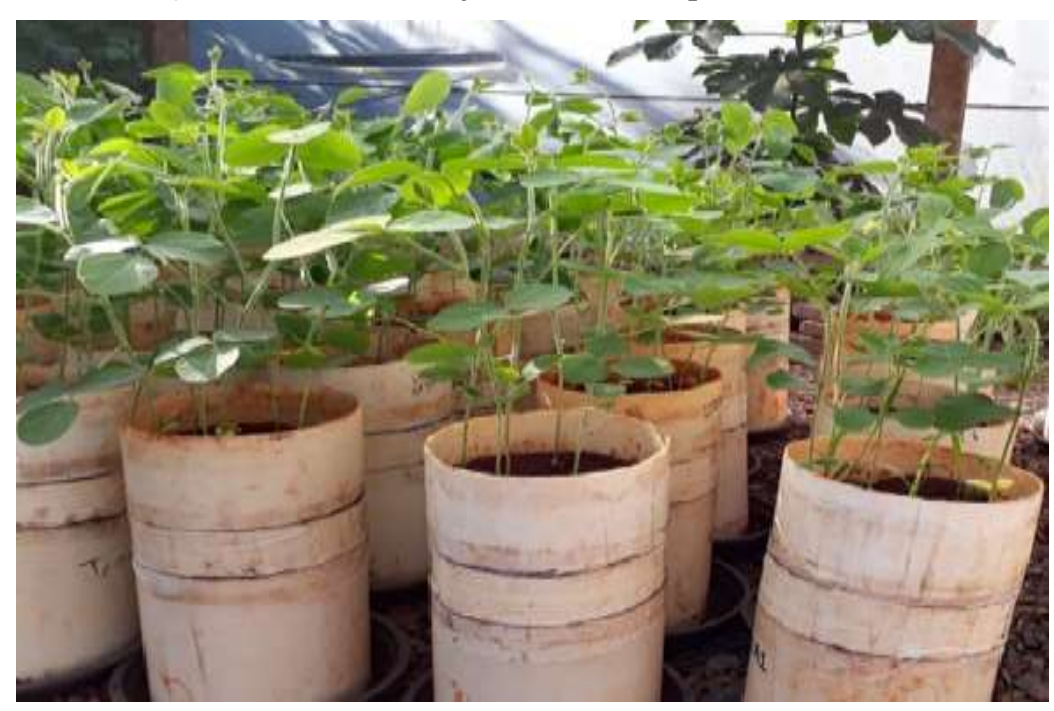

Fonte: Autores.

As folhas moídas foram submetidas à análise de digestão sulfúrica com a utilização de ácido sulfúrico H2SO4, sulfato cobre - CuSO4 e sulfato de potássio - K2SO4. Para determinar nitrogênio, fósforo e potássio outros tipos de ácidos foram utilizados (Embrapa, 2009).

O delineamento experimental utilizado foi inteiramente casualizado com cinco densidades do solo $(1,1 ; 1,2 ; 1,3 ; 1,4$ e 1,5 $\mathrm{Mg} \mathrm{ha}^{-1}$ ) e cinco repetições. Os resultados foram submetidos à análise de variância (ANOVA) ao nível de $5 \%$ de 
significância $(\mathrm{p}<0,05)$ e análise de regressão.

\section{Resultados e Discussão}

As concentrações de N, Ca e S da parte aérea da soja não foram afetadas ( $p>0.05$ ) pelos níveis de compactação do solo (Figura 2A, D e F). Em contrapartida, foi observado acréscimo nos teores de P e K (Figura 2B e C) e redução nos teores de Mg (Figura 2 E) com o aumento da compactação do solo.

Figura 2. Teor de N (A), P (B), K (C), Ca (D), Mg (E) e S (F) de trifólios de soja em função da compactação do solo. * significativo a 5\% de significância pelo teste $\mathrm{F}$. ns=não significativo ao nível de 5\% de significância pelo teste $\mathrm{F}$.
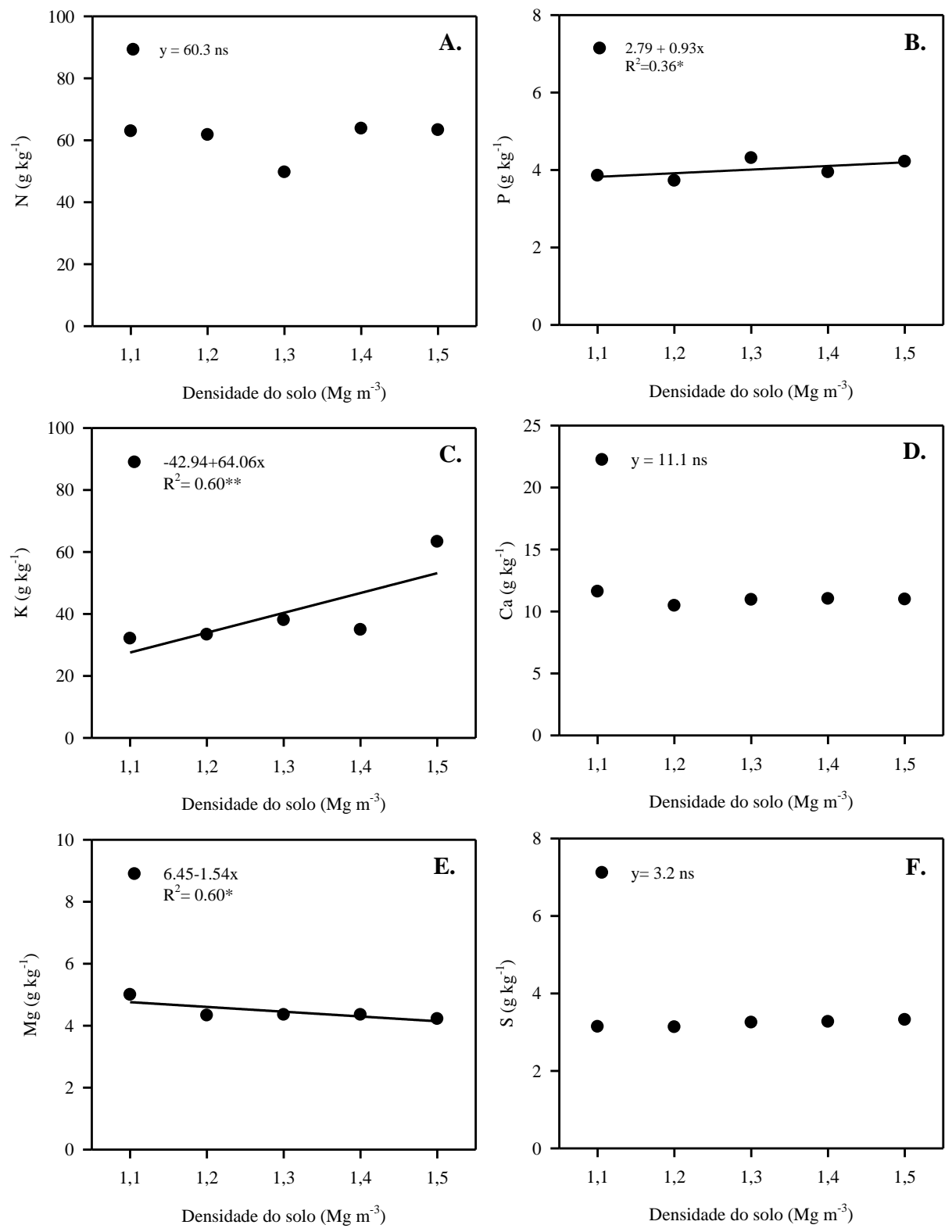

Fonte: Autores.

A compactação do solo induziu alterações na absorção e concentração de $\mathrm{P}, \mathrm{K}$ e Mg nas plantas de soja no estádio R1 (Figura 2). A compactação do solo reduz a porosidade do solo e afeta a dinâmica de água no solo, que é o meio pelo qual os nutrientes são absorvidos e disponibilizados para o metabolismo vegetal. $\mathrm{O}$ aumento do teor de $\mathrm{K}$ com o aumento da 
compactação do solo pode ser explicado pelo aumento da concentração de raízes no anel superior, o que resulta em maior absorção pelos mecanismos de contato, interceptação e difusão. Além disto, pode acorrer menor lixiviação de K nos maiores graus de compactação (Alves et al., 2001).

Em relação ao P, Alves et al. (2001) observaram que a concentração de $\mathrm{P}$ aumentou até a densidade de $1,39 \mathrm{Mg} \mathrm{m}^{-3}$, o que pode ser devido ao maior contato solo e raiz, com observado no presente estudo. O maior contato entre solo e raiz proporciona maior absorção de fósforo. Como este nutriente se move por difusão, possui baixa mobilidade no solo, explicando o teor crescente de concentração foliar no capim-piatã com o aumento dos níveis de compactação observado por Cabral et al. (2012). Corrêa et al. (2001), verificaram aumento nos teores de S, K, N e redução de Zn, B, Fe, Ca e Mg na cana-de-açúcar, à medida que a compactação aumentou. Alves et al. (2001) verificaram que nutrientes mais móveis no solo, como N, K e S, sujeitos à lixiviação, tiveram suas concentrações aumentadas na massa seca da parte aérea do feijoeiro com o aumento da compactação do solo.

A concentração de Mg nas folhas de soja diminuiu significativamente com o aumento da compactação do solo (Figura 2E). A compactação afeta a taxa de infiltração e o fluxo de água no solo (Zambrana et al., 2010), afetando o contato íon-raiz. Com isso, os nutrientes absorvidos por fluxo de massa, como Mg são influenciados (Alves et al., 2003; Cabral et al., 2012).

A qualidade da porosidade do solo afeta negativamente a disponibilidade de nutrientes para as plantas, visto que os mecanismos de fluxo de massa e difusão, responsáveis pelo transporte de nutrientes até as raízes, são processos dependentes da estrutura do solo (Costa et al., 2009); além de alterar os mecanismos pelos quais os nutrientes são transportados no solo, a compactação também pode afetar a quantidade de nutrientes disponíveis (Medeiros et al., 2005).

\section{Conclusão}

A compactação do solo induziu alterações na absorção e concentração de $\mathrm{P}, \mathrm{K}$ e $\mathrm{Mg}$ nas plantas de soja. Foi observado acréscimo nos teores de $\mathrm{P}$ e $\mathrm{K}$ e redução no teor de $\mathrm{Mg}$ com o aumento da compactação.

\section{Referências}

Alves, V. G., Andrade, M. J. B. D., Corrêa, J. B. D., Moraes, A. R. D., \& Silva, M. V. D. (2003). Concentração de macronutrientes na parte aérea do feijoeiro (Phaseolus vulgaris L.) em função da compactação e classes de solos. Ciência e Agrotecnologia, 27, 44-53.

Bareta Junior, E, da Silva, A. A. P., Sens, T. M. Z. G., Colecha, K., Rampim, L., \& Pott, C. A. (2021). Soil physical properties in variable levels of soil compaction. Research, Society and Development, 10(2), e2341028686-e2341028686.

Bergamin, A. C., Vitorino, A. C. T., Franchini, J. C., Souza, C. M. A. D., \& Souza, F. R. D. (2010). Compactação em um Latossolo Vermelho distroférrico e suas relações com o crescimento radicular do milho. Revista Brasileira de Ciência do Solo, 34, 681-691.

Beutler, A. N., \& Centurion, J. F. (2003). Efeito do conteúdo de água e da compactação do solo na produção de soja. Pesquisa Agropecuária Brasileira, 38, $849-856$.

Cabral, C. E., Bonfim-Silva, E. M., Bonelli, E. A., da Silva, T. J., Cabral, C. H., \& Scaramuzza, W. L. (2012). Compactação do solo e macronutrientes primários na Brachiaria brizantha cv. Piatã e Panicum maximum cv. Mombaça. Revista Brasileira de Engenharia Agrícola e Ambiental, $16,362-367$.

Caviglione, J. H., Kiihl, L. R. B., Caramori, P. H., Oliveira, D., Galdino, J., Borrozino, E., \& Pugsley, L. (2000). Cartas climáticas do Estado do Paraná. Londrina: Iapar, 1.

Corrêa, J. B. D., Andrade, L. A., Dias Junior, M. D. S., \& Alves, V. G. (2001). Influência da compactação na concentração de nutrientes da parte aérea da cana-de-açúcar, em três tipos de solos. Revista STAB Açúcar, Álcool e Subprodutos, 19, 34-37.

Costa, J. P., Barros, N. F. D., Bastos, A. L., \& Albuquerque, A. W. D. (2009). Fluxo difusivo de potássio em solos sob diferentes níveis de umidade e de compactação. Revista Brasileira de Engenharia Agrícola e Ambiental, 13, 56-62.

Empresa Brasileira de Pesquisa Agropecuária. (1999). Manual de análises químicas de solos, plantas e fertilizantes. 2, 193-198, Brasília.

Queiroz-Voltan, R. B., Nogueira, S. D. S. S., \& Miranda, M. A. C. D. (2000). Aspectos da estrutura da raiz e do desenvolvimento de plantas de soja em solos compactados. Pesquisa Agropecuária Brasileira, 35, 929-938. 
Research, Society and Development, v. 10, n. 16, e43101623515, 2021

(CC BY 4.0) | ISSN 2525-3409 | DOI: http://dx.doi.org/10.33448/rsd-v10i16.23515

Medeiros, R. D. D., Soares, A. A., \& Guimarães, R. M. (2005). Compactação do solo e manejo da água. I: Efeitos sobre a absorção de N, P, K, massa seca de raízes e parte aérea de plantas de arroz. Ciência e Agrotecnologia, 29, 940-947.

Silva, R. H. D., \& Rosolem, C. A. (2002). Crescimento radicular de soja em razão da sucessão de cultivos e da compactação do solo. Pesquisa Agropecuária Brasileira, 37, 855-860.

Ren, L., Nest, T. V., Ruysschaert, G., D’Hose, T., \& Cornelis, W. M. (2019). Short-term effects of cover crops and tillage methods on soil physical properties and maize growth in a sandy loam soil. Soil and Tillage Research, 192, 76-86.

Silva, V. A., Marchi, G., Guilherme, L. R. G., Lima, J. M. D., Nogueira, F. D., \& Guimarães, P. T. G. (2008). Kinetics of K release from soils of Brazilian coffee regions: effect of organic acids. Revista Brasileira de Ciência do Solo, 32(2), 533-540.

Spliethoff, J., Pott, C. A., Rampim, L., Watzlawick, L. F., \& Jadoski, S. O. (2020). Limites de compactação do solo para Ilex paraguariensis. Research, Society and Development, 9(5), e23953101-e23953101.

Tokura, L. K., Secco, D., Júnior, L. A. Z., Siqueira, J. A. C., Alovisi, A. M. T., Barison, A., \& Zin, Z. (2021). Use of cover crops in Oxisol and its effects on yield and soybean oil content. Research, Society and Development, 10(12), e353101220514-e353101220514.

Valadão, F. C. D. A., Weber, O. L. D. S., Valadão, D. D., Scapinelli, A., Deina, F. R., \& Bianchini, A. (2015). Adubação fosfatada e compactação do solo: sistema radicular da soja e do milho e atributos físicos do solo. Revista Brasileira de Ciência do Solo, 39, 243-255.

Zambrana, M. O. D., Ruiz, H. A., da Silva, T. C. A., Neves, J. C. L., Corrêa, G. F., \& Eraso, M. H. R. (2010). A compactação de três materiais de solo, na redução da condutividade hidráulica, porosidade do solo e matéria seca de raiz nas culturas de soja e caupi. Revista de Ciencias Agrícolas, 27(1), 74-84. 